Dr. Hemmant asked about the cause of leucorrhoea in pregnant women. He thought it must be largely congestive. The mucus was very viscid and was of the pre-menstrual type. It was probably very largely increased by the congestion of the cervix which would increase the gland activity. There was so much hormonal activity during pregnancy that it could be connected with that. The oestral level rose during the three weeks before labour and sometimes increased leucorrhoea could be traced during that time, but he did not have any evidence as to this.

Dr. Mascall asked whether the discharge was due to inereased infection in the vaginal vault. This would be attributing to the cervix an almost conscious bacteriological activity which it hardly possessed. He thought the cervix was not infected in such cases because of the bactericidal effect of the mucus.

Col. Campbell had found excessive leucorrhoea in members of the Auxiliary Territorial Service who were suffering from temporary amenorrhoea. He thought they probably got amenorrhoea because of change of occupation, quite a common oscurrence in young women. The speaker did not quite understand whether Col. Campbell was making any association between the nongonococcal male urethritis and the leucorrhoea of such young people. Was it that they had a non-gonococcal discharge from coitus?

Col. Campbell said that that seemed to be the position.

Mr. Bourne asked whether or not it was trichomoniasis.

Col. Campbell thought it apparently was not. He wondered whether or not the alteration of hydrogen-ion concentration in the oestrus was sufficient to set up a male urethritis.

Mr. Bourne said he had heard that suggestion made on other occasions. He hardly thought it possible, but he had difficulty in understanding how the acid vagina, even at 4 or 3.5 , could apply to or be operative upon the mucous membrane of the male urethra.

Dr. Batchelor raised a point which he had often wished to raise himself, that is, was a gonococcal cervix. infection as common as was thought? Did anyone really now think of the gonococci lurking in hiding in the branched glands? How was it that they could never see them if they were there? Must they not keep an irritative leucocytic infiltration around the glands? He often found it difficult to believe that the cervix could harbour the gonococcus for so long-this was probably heretical! Dr. Doble asked if seborrhoea had been noticed in such cases; emphatically yes. Seborrhoea was a very common condition, accompanied by a greasy scalp, in such young people. With regard to alkalis by mouth the acid in the vagina was a condition outside the body. Most people thought that the acid was produced by the action on the glycogen and Mr. Bourne did not see how alkalizing the alimentary tract could have any influence on the vaginal $p \mathrm{H}$. It was worth rying, however.

Dr. Price had asked a question regarding that very difficult procedure of taking a sterile swab from the cervical canal. If there was much material in the cervix the speaker squeezed it out with the ring forceps after sterilizing chemically as carefully as possible the exposed cervix and the external os. If he could get the fluid squeezed out he took it on the forceps, if he could not he put a swab into the canal and took the utmost care in cleaning it. He thought that the absence of culture indicated that it could be done cleanly, although not always.

Major Prebble asked if he had seen vulvovaginitis due to trichomonas infection ; he could not say that he had and he did not know anyone who had. All believed that vulvovaginitis could be due to other organisms than gonococcus but whether it was due to trichomonas he did not know.

\title{
TRAUMATIC ORCHITIS AND EPIDIDYMITIS
}

\author{
By A. E. W. McLACHLAN, M.B., Ch.B., F.R.S.Ed., D.P.H. \\ Clinical Medical Officer, Joint Committee's Clinic and Medical Officer in Charge \\ of Venereal Diseases, Newcastle General Hospital
}

The rarity of serious testicular lesions supervening on trauma alone, as opposed to the frequent influence of trauma or strain in the localizing in the testis or epididymis of a prostato-vesicular or haematogenous infection, or in reviving a pre-existing lesion, has long been recognized. M. B. Wesson (1928) aptly expresses the opinion of most clinicians in his title " " Traumatic Orchitis ': a Misnomer." In a survey of the literature and in an analysis of seventy cases originally diagnosed as traumatic orchitis, Wesson found that a traumatic aetiology could be sustained in only three cases-two of haematocele and one of torsion of the testis. The infrequency of traumatic lesions is indicated by the fact that since 1929 only one case of orchitis and two of epididymitis, in which the primary aetiological factor was undoubtedly traumatic, have been recognized, by exclusion of all other possibilities, from among the miscellany of intrascrotal lesions met with in venereal disease clinic practice. In many of the cases direct or indirect trauma or strain has been advanced by the patient as the cause of such varied conditions as gonococcal, non-gonococcal, tuberculous or syphilitic epididymitis, gummatous 
orchitis, hydrocele, peri-urethral abscess, malignant disease of the testis or thrombosis of a varicocele.

\section{Traumatic orchitis with supervening fungus testis}

Case (1) $M 4190 / W . L$.-A lorry driver, aged twenty-six, who had been married for five years reported to hospital on January 3rd, 1931. His case was referred as gonococcal epididymitis and extra-marital exposure was denied. Twelve days earlier he had sustained direct injury to the right testicle while loading a heavy box on to a lorry. He felt immediate nausea, faintness and acute pain at the site of injury but all became easier after several minutes. Despite persistence of some nausea and local pain he completed the journey. After several hours the pain gradually exacerbated and when he went to bed the man noticed a slight swelling and marked tenderness of the testicle and discoloration of the scrotum. After three days in bed, during which time the pain remained constant but the swelling did not noticeably increase, the patient sought advice and was told to syringe with potassium permanganate and was given a bottle of medicine. The pain and swelling persisted and the patient reported to the clinic on the twelfth day after injury.

On examination there was a tender, slightly inflamed, boggy swelling, with rather ill defined margins, approximately two and a half inches in diameter on the antero-lateral aspect of the scrotum. There seemed to be a softening in the centre and the scrotum was adherent to the body of the underlying testicle. The epididymis was normal to palpation but the body of the testis, which was difficult to palpate because of the swollen scrotum, appeared tense, tender and slightly enlarged. The prostate and vesicles were normal. There was not any microscopic, cultural or serological evidence of gonorrhoea or syphilis. (When first seen the patient had a slight chemical urethritis from syringing but this settled down rapidly with cessation of treatment.) The patient refused to go to hospital, so rest, support and mildly antiseptic dressings were advised. He reported again on the sixteenth day when the scrotal swelling was one and a half inches in diameter, the central softening more marked, and the body of the testis smaller and less tender. There was slight pyrexia.

The patient was admitted to hospital on the twenty-first day with his condition unaltered. Despite rest in bed and protective dressings, the skin of the involved area of the scrotum had gradually broken down and protrusion of the testis had commenced. Herniation continued in spite of the application of plaster strapping and by the fifty-second day orchidectomy was completed under a general anaesthesia. Subsequent progress was uneventful. The operation specimen showed marked herniation of the body of the testis with large areas of necrobiosis. The tunica albuginea was absent over the area of herniation. The epididymis was normal. A section did not show any evidence of tuberculosis, malignant disease or syphilis. Pathologically the sequence of the condition would appear to be (1) injury of scrotum and testis with rupture of the tunica albuginea, (2) haematoma of

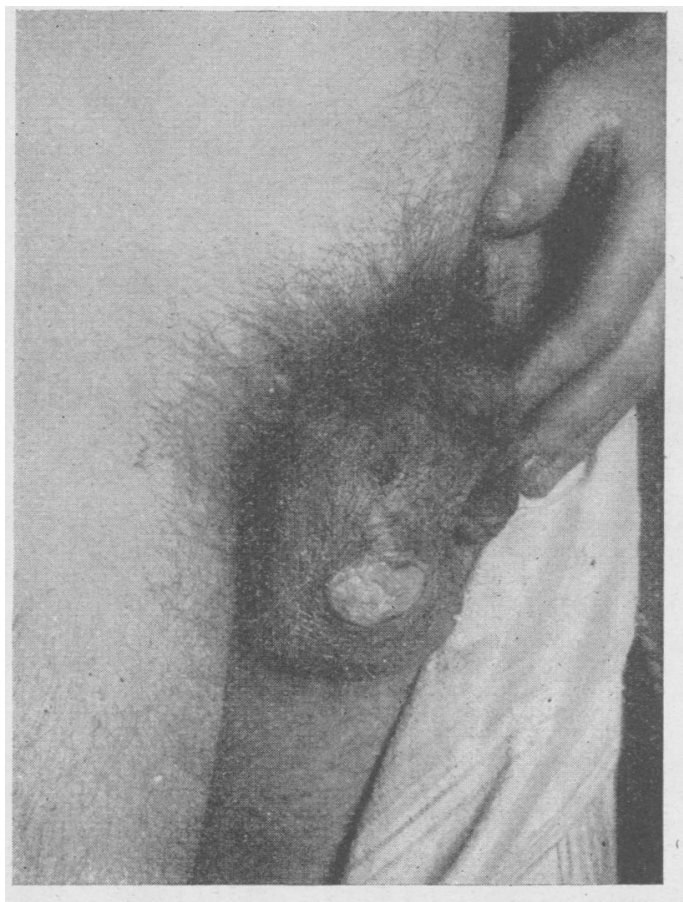

Fig. 1. Traumatic orchitis and scrotal fungus scrotum and of tunica vaginalis, (3) pyogenic infection, (4) herniation of the testicle.

The causation of fungus testis. -This may be (1) traumatic, due to laceration of scrotum with prolapse of testis; to infection of haematoma of scrotum or of tunica vaginalis ; to scrotal sloughing following extravasation of urine ; or, it may be caused by puncture, or application of leeches to inflammatory epididymiti, or hydrocele; (2) Inflammatory, due to tuberculous epididymitis ; gumma of scrotum, tunica vaginalis, or testis ; to typhus, cellulitis of scrotum, erysipelas; to extention of phagedenic gangrene from penile sore. (3) Neoplastic, due to "Fungus haematodes"new growth of testis, etc. fungating through scrotum.

Fungus testis may be (1) superficial in which the protrusion of granulations arises on the outer aspect of the tunica vaginalis or 
tunica albuginea, (2) deep glandular or parenchymatous in which the prolapse of glandular tissue is through the tunica albuginea.

Treatment of traumatic orchitis with fungus testis. - The chief methods of treatment are (1) protective dressings ; (2) strapping to prevent further extrusion when herniation is impending; (3) when extrusion is marked (a) Syme's operation, or, (b) orchidectomy. In Syme's operation an elliptical incision removes the ring of tissue tightly constricting the hernia. The soft parts are carefully dissected, the hernia is dusted with iodoform or sulphonamide powder and reposited in the scrotum which is then apposed over it with as little tension as possible. The danger consists in the cutting through of the stitches. The use of the operation is limited to superficial or early glandular cases. In cases in which there is much damage to the substance of the testis, orchidectomy should be performed.

\section{Contusions of the testicle}

C. E. Monod and O. Terrillon (1889) classify contusions of the testicle as of three degrees: (1) those characterized by minute disseminated capillary haemorrhages in the connective tissue lying between the seminal tubules and the convolutions of the epididymis ; (2) larger effusions varying in size from that of a pea to that of a cherry, associated with laceration of the tubules; (3) rupture of tunica albuginea and formation of acute haematocele.

Béraud (1851) considers that the arrangement of the vessels in a close network on the inner aspect of the tunica albuginea may tend to favour extravasation of blood in this area. E. W. White and R. B. Gaines (1937) state that simple contusions of the testicle rupture small vessels with resulting haemorrhagic points. The pressure of the tunica vaginalis controls the bleeding. There may

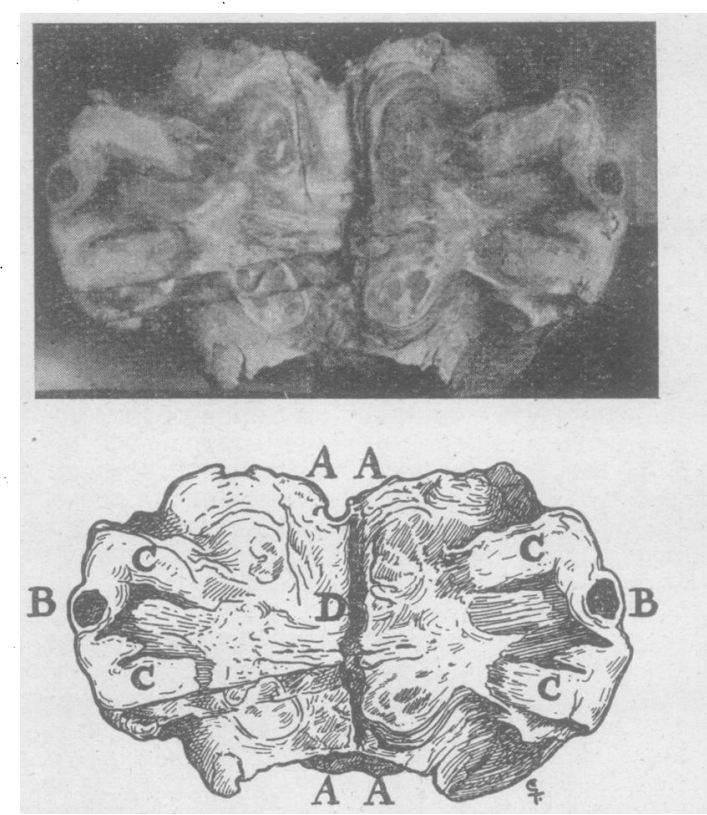

Fig. 2. Section of herniated testicle in Case (1), $A A A A$ skin edges, $B B$ herniated portion of testis, CCCC areas of necrobiosis, $D$ healthy epidydimis. be oedema and the testicle may become enlarged and hard. Fibrosis may follow at the points of injury. If there is extensive cicatricial contracture there may be eventual atrophy of the testicle.

\section{Intratesticular haematocele}

T. Bryant (1888) records two cases of intratesticular haematocele. One case, after injury to the patient on the preceding day, did not present any external signs of injury to the scrotum. The painful testicle was, however, larger than its fellow, the enlargement being in the body of the gland. On careful examination a tense tender point was detected. The introduction of a fine trocar and cannula into the above-mentioned spot freed a jet of blood or blood-strained serum. All pain then ceased and a rapid convalescence followed. The second case was one of intratesticular haemorrhage and haematocele of the tunica vaginalis following evacuation of a hydrocele.

J. W. Churchman (1905) records a case of atrophy of the testis after injury. A man, aged nineteen, had received eleven months previously a kick in the scrotum, which was followed by redness and swelling of the left testicle. The swelling soon went down and atrophy supervened, the testicle gradually shrinking 
until it reached the size of a hazel nut. A section of the testicle showed a good many normal tubules and a few atrophied ones. There was not any connective tissue increase. The vas and epididymis were patent but a good pathological explanation was not found for the atrophy.

Three examples of herniation of the epididymis or body of the testis have been observed after incision or aspiration of a suppurative epididymitis. In these cases spontaneous reposition occurred, with firm healing of the scrotum later.

\section{Traumatic epididymitis}

Two cases in which the injury was localized to the epididymis have been met with.

Case (2) 624/W.L.-A carpenter, aged twenty-six, who had been married for ten years, reported to the clinic on April 26th, 1935 and complained of a swelling of the testicle of three days' duration. He denied extra-marital exposure and a previous history of gonorrhoea or syphilis. He had not noticed any discharge and did not complain of urinary symptoms. He gave a history of "catching" the testicle when his foot slipped on mounting his bicycle eleven days before. There had been temporary nausea and severe pain which had passed off after a few minutes, but the man had been able to walk half a mile home. The next day he had noticed a certain tenderness and bruising of the scrotum. On examination a urethral discharge was not noted, the urine was clear and the prostate normal. Smears, blood W.R. and Gc.F.T. were all negative. There was a swelling the size of a large cherry in the upper pole and extending towards the middle of the epididymis. The swelling was slightly tender and there was a suggestion of fluctuation. Four days later, the patient, who had been advised to apply a protective dressing and a suspensory bandage, reported with a sinus discharging through the upper postero-lateral aspect of the scrotum opposite the upper pole of the right epididymis. Scrapings from the sinus were sent for examination for tubercle and other organisms, but only a scanty growth of Staphylococcus albus was found: Discharge from the sinus continued for approximately a week while gradually ceasing. Healing resulted in a small nodule in the upper pole of the epididymis discrete from the skin. When examined one month later the nodule had shrunk in size to that of a pea. The pathology underlying the case would appear to be that of a small haematocele in the substance of the epididymis followed by secondary infection and abscess formation.

Case (3) M6/N.-A young unmarried man, aged twenty-six, reported on January 4th, 1938 and gave a history of uncomplicated gonorrhoea five years previously. The disease had been adequately treated and there had not been any signs or symptoms since. Three days before reporting the patient had injured a testicle on the saddle when dismounting from his bicycle. He had felt immediate pain but nausea was not severe. Later, he noticed discoloration of the scrotum and a painful nodule in the left testicle. On examination, discoloration of the scrotum from blood extravasation was noted ; in the upper pole of the left epididymis was a tense tender globoid swelling the size of a large cherry. There was not any urethral discharge, the urine was clear, the prostate and vesicles normal, and smears did not show any pus or gonococci. The blood W.R. and Gc.F.T. gave negative results. Under expectant measures' the pain gradually disappeared and the swelling gradually resolved to a small fibrous nodule.

That the original lesions in cases (2) and (3) were in all probability haematomas, infected in the first case, and remaining uninfected in the second, is supported by the observation of W. H. A. Jacobson (1893). Jacobson describes an operation specimen of haematoma of the epididymis associated with a hydrocele. " . . . In the substance of the epididymis was a cavity with a circumference the size of half-a-crown, and enormously thick walls, fibro-cartilaginous in appearance, and enclosing pultaceous, grumous contents, consisting of altered blood ....'

\section{Summary and conclusions}

One case of traumatic orchitis, with supervening fungus testis necessitating orchidectomy, and two cases of haematocele of the epididymis are described.

The infrequency of the conditions in clinical practice is emphasized.

Possible causation and treatment are briefly outlined.

\section{REFERENCES}

Béraud (1851) Arch. gén. Méd., 1, 281.

Bryant, T. (1888) Lancet, 1, 1231.

Churchman, J. W. (1905) Johns Hopk. Hosp. Bull., 16, 265.

Jacobson, W. H. A. (1893) Diseases of the Male Organs of Generation, London, p. 243.

Monod, C. E., and Terrillon, O. (1889) Traité des maladies du testicle et de ses annexes, Paris.

Wesson, M. B. (1928) J. Amer. med. Ass., 91, 1857.

White, E. W., and Gaines, R. B. (1937) J. Amer. med. Ass., 108, 1227. 